\title{
Necrotizing Pneumonia in the Community
}

\author{
Hiroshi Otera ${ }^{1}$, Go Yamamoto ${ }^{2}$, Kiyofumi Ohkusu ${ }^{3}$, Haruka Kozuki ${ }^{4}$, \\ Kimio Hashimoto ${ }^{5}$ and Kimihide Tada ${ }^{1}$
}

\begin{abstract}
A 62-year-old man presented with general fatigue. He was diagnosed with septic shock and severe pneumonia. The sputum at admission yielded methicillin-sensitive Staphylococcus aureus (MSSA) strain and methicillin-resistant $S$. aureus (MRSA) strain. Despite antibiotic treatment, he did not improve. A chest computed tomography (CT) revealed multilobar cavity lesions. Only MRSA strain was confirmed at that time. We diagnosed him with necrotizing pneumonia. Despite treatment with vancomycin, his pneumonia worsened and he died. At autopsy, many gram-positive cocci were observed in the lungs. The clinical presentation of our patient was different from typical CA-MRSA-mediated necrotizing pneumonia.
\end{abstract}

Key words: necrotizing pneumonia, methicillin-resistant Staphylococcus aureus, Panton-Valentine leukocidin

(Intern Med 51: 2463-2467, 2012)

(DOI: 10.2169/internalmedicine.51.7626)

\section{Introduction}

MRSA has traditionally been considered a health careassociated pathogen in patients with established risk factors such as a recent hospitalisation or surgery, residence in a health care facility, treatment by dialysis or the presence of indwelling devices. However, MRSA has also been observed in patients without established risk factors. Therefore, we must consider CA-MRSA and health-care-associated MRSA infections separately (1). CA-MRSA patients are defined as those without documented health care risk factors: 1) the presence of an invasive device at the time of admission; 2) history of MRSA infection or colonization; 3) history of surgery, hospitalization, dialysis, or residence in a long-term care facility in the 12 months preceding culture date (2). Currently, CA-MRSA is mainly associated with skin, soft tissue infections in healthy young persons in the community and to a lesser extent with community-acquired severe pneumonia (3). This type of pneumonia is called necrotizing pneumonia. Since the late 1990s, the number of reports of necrotizing pneumonia due to CA-MRSA has increased in the US (3) and France (4). Necrotizing pneumonia occurs in young, previously healthy adults with a previous episode of an influenza-like illness that is characterized by severe respiratory symptoms, hemoptysis, a high fever, leukopenia, a high CRP level and hypotension. The feature of necrotizing pneumonia is liquefaction and cavitation lung tissue pathologically and is typically multilobar small cavitating alveolar infiltrates as shown on a chest radiograph $(3,5,6)$. The differential diagnosis of cavitary pneumonia is lung abscess. Most lung abscesses develop after aspiration of oral secretions, and therefore occur more commonly in elderly and neurological patients. Lung abscess is characteristic of solitary cavitation surrounded by a well-defined enhancing rim, and may contain an air-fluid level. Not only antibiotics but also percutaneous drainage may be required for the treatment of large abscess. CA-MRSA-mediated necrotizing pneumonia has recently been the focus of attention because it has an epidemiological association with the molecular characteristics of CA-MRSA. Necrotizing pneumonia caused by CA-MRSA has been previously associated with PantonValentine leukocidin (PVL). PVL is a staphylococcal toxin that can induce tissue necrosis (5). CA-MRSA typically harbours the PVL genes. However, the presentation of the patient described herein was necrotizing pneumonia in the community, and different from the typical clinical presentation and the usual laboratory findings of community-

\footnotetext{
${ }^{1}$ Department of Respiratory Medicine, Nishi-Kobe Medical Center, Japan, ${ }^{2}$ Department of Clinical Laboratory Medicine, Nishi-Kobe Medical Center, Japan, ${ }^{3}$ Department of Microbiology, Gifu University Graduate School of Medicine, Japan, ${ }^{4}$ Department of Psychiatry, Nishi-Kobe Medical Center, Japan and ${ }^{5}$ Department of Pathology, Nishi-Kobe Medical Center, Japan

Received for publication March 1, 2012; Accepted for publication May 20, 2012

Correspondence to Dr. Hiroshi Otera, h.otera@onh.go.jp
} 


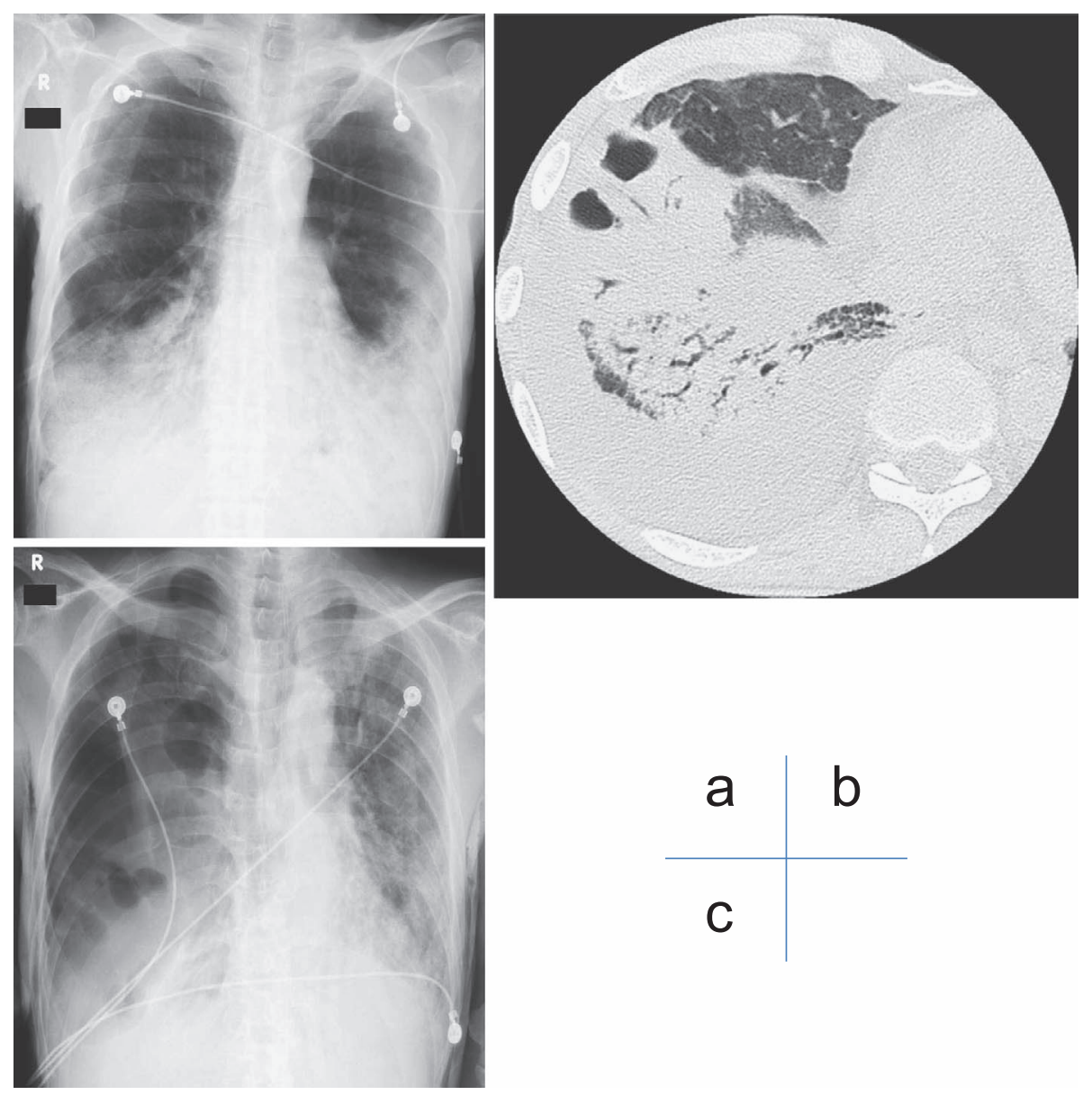

Figure 1. (a) Portable chest radiograph taken on the day of admission to the emergency department. The radiograph showed non-homogenous pulmonary infiltrates with air bronchograms in both sides in the middle to lower fields. (b) High-resolution computed tomography images of the right lung were obtained on day 10 of hospitalization. HRCT showed consolidation with air bronchograms and an increased area of cavitation in the right lower lobe and pleural effusion. (c) Portable chest radiograph taken on day 15 of hospitalisation showing a pneumothorax in the right lung.

acquired pneumonia (CAP) caused by PVL-positive MRSA.

\section{Case Report}

In January 2009, a 62-year-old Japanese man presented at our emergency department with general fatigue. The patient had bipolar disorder. He was an outpatient of the psychiatry department and regularly consulted a psychiatrist approximately once every two months. He had not been receiving antibiotics in general, of course, had not received at several weeks of hospitalization. The patient's last hospitalization was three years prior to this hospital admittance for an operation to treat prostatic hypertrophy. He had been unemployed for several years. There were no family contacts that work in health care. The patient was a former smoker with a history of one pack a day for 40 years and no known lung disease. He had no previous history of MRSA infection.

At admission, he was drowsy, but was easily aroused. A few days before hospital admittance, the patient felt ill with coughing and a high fever without hemoptysis. On admission, the patient's body temperature was $32.8^{\circ} \mathrm{C}$, his blood pressure was $62 / 30 \mathrm{~mm} \mathrm{Hg}$, his pulse rate was 66 beats/min, and his percutaneous oxygen saturation was $89 \%$ in room air. Physical examination revealed coarse crackles over the posterior lower portions of both lungs. The patient did not display any skin lesions. A rapid influenza diagnostic test was negative. Tests for urinary antigens of Legionella pneumophila and Streptococcus pneumoniae were negative. A chest radiograph showed bilateral patchy infiltrates in both lower lung fields (Fig. 1a). The level of his consciousness decreased. The patient's condition was diagnosed as septic shock, with severe pneumonia and meningitis suspected. We intravenously administered $2 \mathrm{~g}$ meropenem and a norepinephrine infusion and provided oxygen. The patient's white blood cell count was 21,400 cells $/ \mathrm{mm}^{3}$. The C-reactive protein (CRP) level was $20.6 \mathrm{mg} / \mathrm{dL}$, the blood urea nitrogen level was $58 \mathrm{mg} / \mathrm{dL}$, and the creatinine level was $2.2 \mathrm{mg} / \mathrm{dL}$. In addition, we performed a cerebrospinal fluid examination to confirm or rule out the diagnosis of meningitis, and the results were normal. The patient's sputum, which was obtained at the time of admission by suctioning, was purulent. It revealed gram-positive cocci and neutrophils, and phago- 


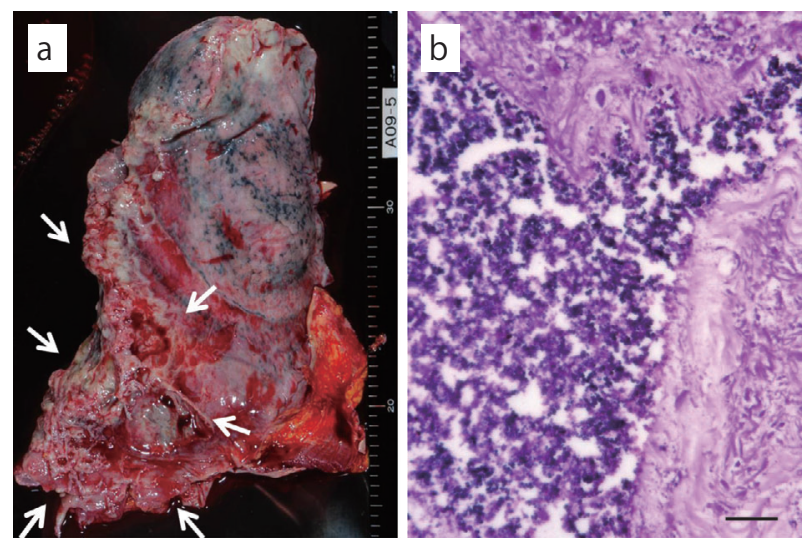

Figure 2. (a) Whole right lung specimen obtained by autopsy. Gross pathological findings revealed that the right lower lobe was predominantly coalesced with the parietal pleura (arrow). (b) Pathological findings of the right lung. The specimen displayed numerous gram-positive cocci (blue stain) and neutrophils that were clustered in the area that was enclosed by the parietal and visceral pleura. (Internal scale bar $=\mathbf{1 0 0} \mu \mathrm{m}$; gram stain.)

cytosis was not observed in Gram stains. A few days later, the culture of the sputum sample revealed MRSA and MSSA. He had negative blood cultures.

After the patient received treatment, the physical and blood analyses did not reveal any improvement. A chest radiograph revealed worsening of the lobar consolidation and the expansion of consolidations. On day 10 of hospitalization, a chest CT was performed, which revealed cavity lesions, multilobar dense infiltrates and bilateral pleural effusion (Fig. 1b). We diagnosed the patient with necrotizing pneumonia. Right pleural effusion was yellowish and turbid, and many gram-positive cocci and neutrophils were found by microscopic examination. The pleural effusion cultures confirmed the presence of MRSA; MSSA was not detected. Upon re-examination of his sputum, MSSA was no longer detected. On hospital day 11, intravenous vancomycin $1 \mathrm{~g}$ twice a day was added to the patient's therapy. On hospital day 15 , a chest radiography showed a pneumothorax and atelectasis of his right lung (Fig. 1c). The patient died 18 days after his admission to the hospital. The post-mortem examination revealed extensive necrotizing pneumonia with large lung abscesses (Fig. 2a). Histology revealed diffuse alveolar damage and many gram-positive cocci in the lungs (Fig. 2b). There was no bleb or emphysematous change.

The $S$. aureus isolates from sputum and pleural effusion were resistant to oxacillin, imipenem/cilastatin, $\beta$-lactam antibiotics, erythromycin and fluoroquinolones but susceptible to vancomycin, trimethoprim-sulfamethoxazole, rifampicin and arbekacin. The isolates were tested for genes encoding selected toxins [staphylococcal enterotoxin (SE) B; exfoliative toxin; PVL; and toxic shock syndrome toxin 1 (TSST1)] by multiplex real-time polymerase chain reaction (PCR) analysis. The MRSA isolates were typed to determine their staphylococcal cassette chromosome mec (SCCmec) type by multiplex PCR targeting the cassette chromosome recombinases (ccr) and mec gene complex (6). Pulsed-field gel electrophoresis (PFGE) and multilocus sequence typing (MLST) were not performed. The MRSA isolate from this patient was negative for PVL and belonged to the group of SCCmec type II strains. This $S$. aureus isolate was positive for TSST-1 and negative for SEB and exfoliative toxin.

\section{Discussion}

MRSA was previously regarded solely as a hospitalacquired pathogen, but it is now emerging as a communityacquired pathogen $(3,7)$. CA-MRSA is an increasing problem in several countries $(3,7,8)$, and it is associated with necrotizing pneumonia (3). PVL has been identified in many previous studies of necrotizing pneumonia. PVL is a staphylococcal toxin with a well-established cytolytic mechanism that specifically targets phagocytic leukocytes (3). However, the role of PVL in necrotizing lesion development is still controversial (9), and is probably due to the species-specific susceptibility of neutrophils to the lytic effects of PVL (10). The prevalence of the PVL genes varies geographically $(3,11)$. MRSA containing the PVL genes comprises $0.1 \%$ of MRSA isolates that have been collected in hospitals in Japan (12). Although there has been no large study conducted in Japan, it is thought that the prevalence of the PVL genes is significantly lower in Japan than in the USA $(11,13)$. Most of the reported Japanese cases of CAMRSA were not severe and included skin infections. However, several studies have reported severe PVL-positive MRSA infections in the community (14).

The present patient required ventilator support based on the observable vital and laboratory data. The radiographic findings revealed that the patient had multilobar infiltrates and cavitation. The isolates from both sputum and pleural effusion were MRSA. These findings suggested necrotizing pneumonia and PVL-positive MRSA infection in the community. However, the patient was older and had had some past diseases, and the clinical progression of the patient's pneumonia was not as rapid as previous cases of CA-MRSA carrying PVL genes. The patient's blood analysis showed leukocytosis. Furthermore, the drug sensitivity pattern and the SCCmec type of the MRSA isolated from sputum and pleural effusion were characteristic of health care-associated MRSA and not CA-MRSA $(3,6)$. This MRSA seems to be the New York/Japan clone. However, this clone cannot be identified since some important genotypes (such as multilocus sequence type and spa type) were not performed. We should have characterized MRSA more carefully. The strain isolated from this patient was a health care-associated type of strain but it caused a CA-MRSA-type syndrome. Additionally, MRSA infection was not suspected, based on the history taken at admission. However, the patient had a prior hospital admission 3 years previously, and we did not culture the anterior nares upon the current admission of the patient. Previously colonized patients with MRSA should be 
considered to be at high risk of continued carriage for at least 4 years (15). This supports the fact that it is difficult to establish the origin of strains causing MRSA infections in the community. Risk factors for the acquisition of strains were related to the health care environment.

Furthermore, the patient's sputum at admission yielded not only MRSA strain but also MSSA strain. After treatment with meropenem, the patient no longer harboured MSSA in his sputum or pleural effusion according to our test results. For this reason, we think that the MSSA infection was successfully treated by antibiotics. However, it is hard to say that only MRSA caused his clinical course from beginning to end. An effect from his early clinical condition from the MSSA cannot be completely eliminated. It is more reasonable to think that the appearance of substituted microbism was caused by antibiotics and it made a translation to MRSA pneumonia. Tomita et al. reported two cases with severe necrotizing pneumonia due to CA-MRSA infection (16). Both of their MRSA isolates were PVL negative. In their discussion, they pointed out reasons for the delay in starting treatment for MRSA; the reasons were that they had a lack of understanding of CA-MRSA and considered the MRSA of their patient's sputum at admission as colonization. In the present case, we also considered such reasons, and did not change meropenem after it became clear that his sputum yielded MRSA at admission. In general, we think of community-associated strains as invading the health care environment rather than the other way around. Although a patient who carries MRSA in the nasal mucosa is a large problem for the prevention of nosocomial infection in the community, the presence of MRSA is often not taken seriously in patients leaving the hospital. There remains the possibility of affecting the bacterial flora of the community environment. Our patient's diagnosis appears not to meet the criteria for CA-MRSA.

TSST-1 is one of the superantigens and suppresses the motility of polymorphonuclear neutrophils by inhibiting the expression of MRSA exoproteins (11). Orii et al. (17) reported a fatal case of necrotizing fasciitis due to a PVLnegative MRSA strain that expressed TSST-1 and suggested that the pathogenesis is the result of a factor other than PVL. Although our patient presented with shock, we did not observe rash, desquamation or high fever. This profile did not fit the established definition of toxic shock syndrome (18) but most likely does fit with the proposed definition of "toxic shock syndrome-like illness" (19). His MRSA isolates had TSST-1. We did not examine the MSSA isolate, so we do not know whether this isolate was PVL and TSST- 1 positive. In addition to TSST-1 and PVL, other toxins (notably alpha-hemolysin) may have influenced his clinical condition.

Although the laboratory data did not support this diagnosis, the patient could have been infected with influenza. The incident occurred during influenza season, and RT-PCR for influenza A and B was not performed. Influenza infection has an important role in MRSA infection (20), and thus complicating effects from influenza infection cannot be completely excluded.

In conclusion, not all MRSA isolates in the community harbour the PVL genes. Various factors influenced the clinical progression of the present case of necrotizing pneumonia. Based on the results of the current study, we concluded that MRSA not involving PVL genes can cause necrotizing pneumonia. It is doubtful whether PVL has an important role in the virulence of CA-MRSA. PVL may be one of the genetic markers for CA-MRSA. Medical personnel must not delay in the diagnosis of necrotizing pneumonia when PVLnegative MRSA isolates are observed in the community. In Japan, the prevalence of PVL-positive MRSA is low, and clinical laboratory testing for PVL genes is rare. Moreover, the rate of methicillin resistance in the causative organisms of CAP in adults is very low (21). Therefore, vancomycin should not be used for empiric therapy in every patient with CAP, especially in Japan, except for usage in a typical case. However, clinicians need to maintain a heightened awareness that CA-MRSA can cause rapidly progressive, necrotizing infections such as pneumonia. Close monitoring, timely modification of empiric antimicrobial coverage, additional testing for PVL or other MRSA- or MSSA-associated toxins, and timely treatment for toxins should be considered as a part of clinical practice.

The authors state that they have no Conflict of Interest (COI).

\section{References}

1. Naimi TS, LeDell KH, Como-Sabetti K, et al. Comparison of community- and health care-associated Methicillin-resistant Staphylococcus aureus infection. JAMA 290: 2976-2984, 2003.

2. Klevens RM, Morrison MA, Nadle J, et al. Active Bacterial Core surveillance (ABCs) MRSA Investigators: Invesive methicillinresistant Staphylococcus aureus infections in the United States. JAMA 298: 1763-1771, 2007.

3. Hidron AI, Low CE, Honig EG, Blumberg HM. Emergence of community-acquired meticillin-resistant Staphylococcus aureus strain USA300 as a cause of necrotising community-onset pneumonia. Lancet Infect Dis 9: 384-392, 2009.

4. Dufour P, Gillet Y, Bes M, et al. Community-acquired methicillinresistant Staphylococcus aureus infections in France: emergence of a single clone that produces Panton-Valentine leukocidin. Clin Infect Dis 35: 819-824, 2002.

5. Gillet Y, Issartel B, Vanhems P, et al. Association between Staphylococcus aureus strains carrying gene for Panton-Valentine leukocidin and highly lethal necrotising pneumonia in young immunocompetent patients. Lancet Infect Dis 359: 753-759, 2002.

6. Que YA, Moreillon P. Staphylococcus aureus (including Staphylococcal toxic shock). In: Mandell, Douglas, and Bennett's Principles and Practice of Infectious Diseases - Expert Consult Premium ed. 7th ed. Mandell GL, Bennett JE, Dolin R, Eds. Churchill Livingstone, Philadelphia, 2010: 2543-2578.

7. Vandenesch F, Naimi T, Enright MC, et al. Community-acquired methicillin-resistant Staphylococcus aureus carrying PantonValentine leukocidin genes: worldwide emergence. Emerg Infect Dis 9: 978-984, 2003.

8. Eady EA, Cove JH. Staphylococcal resistance revisited: community-acquired methicillin resistant Staphylococcus aureus- 
an emerging problem for the management of skin and soft tissue infections. Curr Opin Infect Dis 16: 103-124, 2003. Review.

9. Diep BA, Otto $M$. The role of virulence determinants in community-associated MRSA pathogenesis. Trends Microbiol 16 361-369, 2008.

10. Löffler B, Hussain M, Grundmeier M, et al. Staphylococcus aureus panton-valentine leukocidin is a very potent cytotoxic factor for human neutrophils. PLoS Pathog 6: e1000715, 2010.

11. Yamamoto T, Nishiyama A, Takano T, et al. Community-acquired methicilin-resistant Staphyrococcus aureus: community transmission, pathogenesis, and drug resistance. J Infect Chemother 16: 225-254, 2010.

12. Yamamoto T, Dohmae S, Saito K, et al. Molecular characteristics and in vitro susceptibility to antimicrobial agents, including the des-fluoro(6) quinolone DX-619, of Panton-Valentine leucocidinpositive methicillin-resistant Staphylococcus aureus isolates from the community and hospitals. Antimicrob Agents Chemother 50: 4077-4086, 2006.

13. Hisata K, Kuwahara-Arai K, Yamanoto M, et al. Dissemination of methicillin-resistant staphylococci among healthy Japanese children. J Clin Microbiol 43: 3364-3372, 2005.

14. Ito $T$, Iijima $M$, Fukushima $T$, et al. Pediatric pneumonia death caused by community-acquired Methicillin-resistant Staphylococcus aureus, Japan. Emerg Infect Dis 14: 1312-1314, 2008.
15. Robicsek A, Beaumont JL, Peterson LR. Duration of colonization with Methicillin-Resistant Staphylococcus aureus. Clin Infect Dis 48: 910-913, 2009.

16. Tomita $\mathrm{Y}$, Kawano O, Ichiyasu $\mathrm{H}$, et al. Two cases of severe necrotizing pneumonia caused by community-acquired methicillinresistant Staphylococcus aureus. Nihon Kokyuki Gakkai Zasshi 46: 395-403, 2008 (in Japanese, Abstract in English).

17. Orii KO, Iwao Y, Higuchi W, Takano T, Yamamoto T. Molecular characterization of methicillin-resistant Staphylococcus aureus from a fatal case of necrotizing fasciitis in an extremely low-birthweight infant. Clin Microbiol Infect 16: 289-292, 2010.

18. Tofte RW, Williams DN. Toxic shock syndrome. Evidence of a broad clinical spectrum. JAMA 246: 2163-2167, 1981.

19. Parsonnet J. Case definition of staphylococcal TSS: a proposed revision incorporating laboratory findings. In: International Congress and Syndrome. Arbuthnott J, Furman B, Eds. Royal Society of Medicine Press, London, 1998: 15-16.

20. Hageman JC, Uyeki TM, Francis JS, et al. Severe communityacquired pneumonia due to Staphylococcus aureus, 2003-04 influenza season. Emerg Infect Dis 12: 894-899, 2006.

21. Saito A, Kohno S, Matsushima T, et al. Study Group. Prospective multicenter study of the causative organisms of communityacquired pneumonia in adults in Japan. J Infect Chemother 12: 63-69, 2006.

(C) 2012 The Japanese Society of Internal Medicine http://www.naika.or.jp/imonline/index.html 